\title{
Multidrug resistance-associated protein 1 decreases the concentrations of antiepileptic drugs in cortical extracellular fluid in amygdale kindling rats
}

\author{
Ying-hui $\mathrm{CHEN}^{1,2, *}$, Cui-cui WANG ${ }^{1,2}, \mathrm{Xia}_{\mathrm{XIAO}}^{1,2}, \mathrm{Li} \mathrm{WEI}^{1,2}$, Guoxiong XU $\mathrm{XU}^{3, *}$ \\ ${ }^{1}$ Department of Neurology, Jinshan Hospital, Fudan University, Shanghai 201508, China; ${ }^{2}$ Department of Neurology, Shanghai Medical \\ College, Fudan University, Shanghai 200032, China; ${ }^{3}$ Center Laboratory, Jinshan Hospital, Fudan University, Shanghai 201508, China
}

\begin{abstract}
Aim: To investigate whether multidrug resistance-associated protein 1 (MRP1) was responsible for drug resistence in refractory epilepsy in amygdale kindling rats.

Methods: Rat amygdale kindling was used as a model of refractory epilepsy. The expression of MRP1 mRNA and protein in the brains was examined using RT-PCR and Western blot. MRP1-positive cells in the cortex and hippocampus were studied with immunohistochemical staining. The rats were intraperitoneally injected with phenytoin $(50 \mathrm{mg} / \mathrm{kg})$ or carbamazepine (20 mg/kg), and their concentrations in the cortical extracellular fluid were measured using microdialysis and HPLC. Probenecid, a MRP1 inhibitor (40 mmol/L, $50 \mu \mathrm{L}$ ) was administered through an inflow tube into the cortex 30 min before injection of the antiepileptic drugs.

Results: The expression of MRP1 mRNA and protein was significantly up-regulated in the cortex and hippocampus in amygdale kindling rats compared with the control group. Furthermore, the number of MRP1-positive cells in the cortex and hippocampus was also significantly increased in amygdale kindling rats. Microdialysis studies showed that the concentrations of phenytoin and carbamazepine in the cortical extracellular fluid were significantly decreased in amygdale kindling rats. Pre-administration of probenecid could restore the concentrations back to their control levels.
\end{abstract}

Conclusion: Up-regulation of MRP1 is responsible for the resistance of brain cells to antiepileptic drugs in the amygdale kindling rats.

Keywords: multidrug resistance-associated protein 1; refractory epilepsy; amygdale kindling; antiepileptic drug; phenytoin; carbamazepine; cortex; hippocampus; microdialysis

Acta Pharmacologica Sinica (2013) 34: 473-479; doi: 10.1038/aps.2012.183; published online 11 Mar 2013

\section{Introduction}

Refractory epilepsy is a common neurologic disease that accounts for approximately one-third of epileptic patients with resistance to single or multiple drugs used for antiepileptic treatment ${ }^{[1,2]}$. Clinical studies have shown that if the first antiepileptic drug (AED) prescribed was ineffective, switching to another AED or to a combination of AEDs yielded similar negative results. This outcome may suggest that there are nonspecific mechanisms that reduce the sensitivity and effectiveness of AEDs in the brains of patients with refractory epilepsy, despite the various pharmacological mechanisms of action of AEDs. Similar to the mechanism of chemotherapy drug resistance, the development of refractory epilepsy may be caused by the increased expression of multidrug transporters, which

\footnotetext{
* To whom correspondence should be addressed.

E-mail cyh1973131@163.com (Ying-hui CHEN); guoxiong.xu@fudan.edu.cn (Guoxiong XU)

Received 2012-09-25 Accepted 2012-12-05
}

subsequently leads to the decrease in the concentrations of a variety of AEDs in the cortical extracellular fluid of the brain.

There are several well-known multidrug transporters, including P-glycoprotein (PGP) and multidrug resistanceassociated protein 1 (MRP1), in mammals. MRP1 is the most widely researched multidrug transporters and plays an important role in chemotherapy drug resistance ${ }^{[3,4]}$. In this study, the expression of MRP1 in the brain cells of a refractory epilepsy model was investigated. The effect of the inhibition of MRP1 on the concentrations of two AEDs, phenytoin (PHT) and carbamazepine (CBZ), in the cortical extracellular fluid of the refractory epilepsy model was also evaluated.

\section{Materials and methods} Experimental animals

Eighty male Sprague-Dawley (SD) rats (weighing 250-300 g, 6 weeks old) were purchased from Shanghai Animal Center, Shanghai Medical College of Fudan University (Shanghai, China) and housed in the animal facility for at least $5 \mathrm{~d}$ before 
use. All animal experiments were approved by the Ethics Committee of Animal Care of the Jinshan Hospital according to the Guidelines for Animal Experiments of the Chinese Academy of Medical Sciences. These animals were randomly divided into a normal control group and a group of amygdale kindling that developed refractory epilepsy (referred to as the epilepsy group) for the examination of MRP1 expression (16 rats each). Subjects were separated into a PHT group and a CBZ group for the microdialysis experiment (24 rats each). The PHT and CBZ groups were further divided into three subgroups, eight rats in each sub-group: (1) control group: normal rats were intraperitoneally injected with AEDs (PHT $50 \mathrm{mg} / \mathrm{kg}$ or CBZ 20 mg/kg, Sigma, St Louis, MO, USA); (2) epilepsy group: epileptic rats were intraperitoneally injected with PHT or CBZ; and (3) probenecid group: epileptic rats were administered $50 \mu \mathrm{L}$ probenecid (40 mmol/L, Sigma) at the flow rate of $2.5 \mu \mathrm{L} / \mathrm{min}$ through an inflow tube into the cortex $30 \mathrm{~min}$ before intraperitoneal injection with AEDs.

\section{Generation of the amygdale kindling model with refractory epilepsy}

An amygdale kindling rat model resistant to AEDs was generated based on the well-established protocol ${ }^{[5]}$. The seizure severity was assessed in accordance with the standards of Racine $^{[6]}$ and classified into six stages. A rat with at least three consecutive seizures at stage $\mathrm{V}$ was considered an elicited kindling animal. After successful modeling, experimental animals received intraperitoneal injection of PHT or CBZ and were screened for drug resistance. The model of refractory epilepsy was established by repeated drug administration and then selection based on the changes of after discharge threshold (ADT). Electrode-implanted rats were used as the control group.

\section{Immunohistochemical staining}

Rat brains were sectioned $(4 \mu \mathrm{m})$ and incubated with antiMRP1 primary antibody (Santa Cruz Technologies, Santa Cruz, CA, USA) overnight. Sections were then incubated with a secondary antibody for $2 \mathrm{~h}$. After counterstaining with Haematoxylin solution, MRP1-positive cells were counted and photographed using a light microscope.

\section{RNA extraction and RT-PCR}

Total RNA from the brain tissue was extracted using Trizol reagent (Invitrogen, New York, NY). One microgram of total RNA was reverse-transcribed using a reverse transcription kit (Invitrogen), and the specific gene of interest was then amplified using PCR. The primer sequences were 5'-CCATTCAGGCCGTGTAGAGT-3' (forward) and 5'-TCATGGTTCAGCTTGTCAGG-3' (reverse) for MRP1 (404 bp) and 5'-AACCCTAAGGCCAACCGTGAAAAG-3' (forward) and 5'-TCATGAGGTAGTCTGTCAGGT-3' (reverse) for $\beta$-actin (241 bp). RNA was first denatured at $95^{\circ} \mathrm{C}$ for $2 \mathrm{~min}$. Then, PCR amplification was performed at $94^{\circ} \mathrm{C}$ for $45 \mathrm{~s}, 54^{\circ} \mathrm{C}$ for $45 \mathrm{~s}$, and $72^{\circ} \mathrm{C}$ for $1 \mathrm{~min}$ for 30 cycles, with a final extension for $5 \mathrm{~min}$ at $72^{\circ} \mathrm{C}$. The PCR products were then separated on a 1.5\% agarose gel and analyzed using a gel imaging system (GeneGenius, Syngene Co, Ltd, Cambridge, UK).

\section{Western blotting}

Total protein from brain tissue was extracted. Equal amounts of protein samples were run on SDS-PAGE gels and transferred to PVDF membranes. After blocking with $1 \%$ skim milk in TBS-T at room temperature for 1 hour, the membrane was probed with rabbit anti-rat primary antibody (MRP1 and $\beta$-actin, 1:500 dilution) at $4^{\circ} \mathrm{C}$ overnight and subsequently incubated with horseradish peroxidase-conjugated goat antirabbit secondary antibody (1:2000 dilution) at room temperature for $2 \mathrm{~h}$. Signals were detected using ECL-Plus (Santa Clara, CA, USA) and quantified using a Bio-Rad2000 gel imaging system with QUANTITY ONE software (Bio-Rad Laboratories, Hercules, CA, USA).

\section{Microdialysis}

Microdialysis, a minimally invasive sampling technique, is used to consecutively measure the concentration of a free, unbound analyte in the extracellular fluid of virtually any tissue $^{[7]}$. First, a catheter was implanted. A rat was anesthetized by intraperitoneal injection with $1 \%$ pentobarbital sodium (Sigma) at $40 \mathrm{mg} / \mathrm{kg}$ and the head was fixed in a stereotaxic apparatus (RWD Life Science Co, Ltd, Shenzhen, China). A hole was drilled at the middle cortex of the frontal area, $1 \mathrm{~mm}$ away from the anterior fontanelle. At a 30-degree angle from the vertical line, a catheter was inserted via the hole $3 \mathrm{~mm}$ into the cerebral cortex and then secured with dental cement. The microdialysis experiment was performed at a week post-surgery. After connecting the microdialysis device, the probe, along with the catheter, was inserted into the rat cerebral cortex. The catheter probe, which was entirely immersed in the cortex, consisted of a shaft with a semipermeable fiber membrane at its tip. Second, the recovery rate of the AEDs was calculated prior to each microdialysis experiment to determine the permeability of the drug through a semipermeable membrane. The permeability of the membrane to a drug is specific to individual drug. Briefly, the artificial cerebrospinal fluid with $20 \mu \mathrm{g} / \mathrm{mL}$ of PHT or CBZ was prepared and maintained at $37^{\circ} \mathrm{C}$. The tip of the probe was immersed in the solution and the artificial cerebrospinal fluid was injected using a microperfusion pump (KD100, KD Scientific Inc, Holliston, MA) through the inflow tube, probe, and outflow tube at a constant flow rate of $2.5 \mu \mathrm{L}$ per min for $1 \mathrm{~h}$. Following three consecutive collections of $30 \mu \mathrm{L}$ effluent each, the concentration of the effluent drug was measured. The recovery rate was calculated by dividing the effluent drug concentration by the standard drug concentration. Finally, for microdialysis sampling, $25 \mu \mathrm{L}$ of exchanged dialysate of effluent was collected at $15,30,45,60,90,120,150$, and $180 \mathrm{~min}$ after intraperitoneal injection of $50 \mathrm{mg} / \mathrm{kg}$ PHT or $20 \mathrm{mg} / \mathrm{kg} \mathrm{CBZ}$. Because the permeability of the semipermeable membrane was different for each drug, the actual drug concentration in the cortical extracellular fluid was normalized to the recovery rate: the drug concentration in the cortical extracellular fluid=the drug 
concentration in dialysate/recovery rate.

Measurement of drug concentration by high performance liquid chromatography (HPLC)

In order to monitor the concentrations of drugs in the plasma, 16 normal rats were divided into two PHT and CBZ groups. After intraperitoneal injection of PHT or CBZ, the concentrations of PHT and CBZ in the dialysate and serum were determined by HPLC (MODEL 510, Beijing Syltech Scientific Instrument Co, Ltd, Beijing, China). A sample $(20 \mu \mathrm{L})$ was prepared according to the manufacturer's protocol. The chromatographic conditions were as follows: column ODS C18 (200 mm*4 mm, particle $10 \mu \mathrm{m}$, Lichrosorb RP); the mobile phase: methanol/water (55:45, v:v); flow rate: $1.3 \mathrm{~mL}$ per min; UV wavelength of detection: $210 \mathrm{~nm}$; pressure of pump: 1500 ps; paper running speed: $0.5 \mathrm{~cm}$ per min. The minimum detection concentrations were $0.4 \mu \mathrm{g} / \mathrm{mL}$ for PHT and $0.3 \mu \mathrm{g} / \mathrm{mL}$ for CBZ.

\section{Statistical analysis}

All statistical analyses were carried out using SigmaStat (Chicago, IL, USA). Comparisons between groups were performed using either unpaired Student $t$-tests or a one-way ANOVA followed by a Tukey test. Data are presented as the mean $\pm S E M$ or SD where indicated. Differences are considered significant at values of $P<0.05$.

\section{Results}

MRP1 expression was up-regulated in the cortex and hippocampus of the epileptic brain

To investigate the expression of MRP1, we separated the cortex and hippocampus from the rat brain. The expression of MRP1 mRNA in control and epileptic animals was detected by RT-PCR. We found that the expression of MRP1 mRNA was higher in epileptic brains than in normal brains (Figure 1A). Semi-quantitative analysis of the gel after densitometry showed that the expression of MRP1 mRNA was significantly increased in both the cortex and hippocampus of epileptic animals (Figure $1 \mathrm{~B} ; P<0.05$ vs control, $n=8$ ). However, there was no difference in the expression levels observed between the cortex and hippocampus, in either the control or epileptic groups. This result indicated that the increase in MRP1 mRNA was characteristic of epilepsy, regardless of its distribution in the brain.

This increase was also observed at the protein level. Using an antibody specific to MRP1, the expression of MRP1 protein was found to increase significantly in the epileptic brain (Figure 2A and 2B; $P<0.05$ vs control brain, $n=8$ ). Next, we examined the expression of MRP1 protein in brain cells using immunohistochemistry. MRP1 immunoreactive particles were mainly distributed in the cell membrane, with only some found in the cytoplasm. In electrode-implanted control brains, there was a small number of MRP1-positive cells in the cortex. These cells were identified as neurons and glial cells, as the neurons showed a pyramid-like shape, whereas the glial cells showed triangular, oval, or multilateral irregular shapes (Fig-

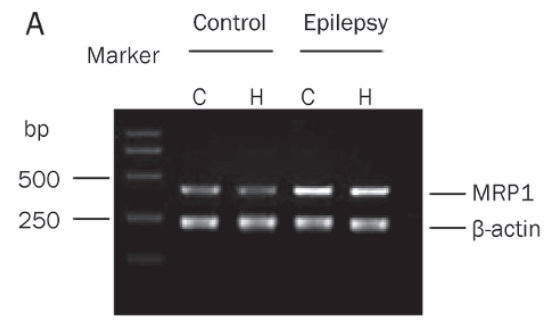

B

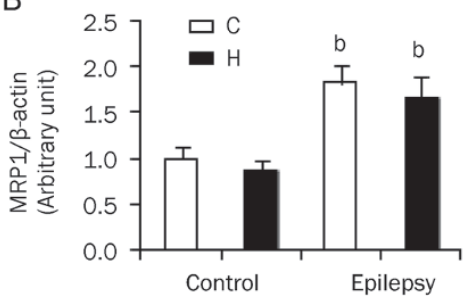

Figure 1. MRP1 mRNA expression in the brain. MRP1 mRNA was detected by RT-PCR. RNA was extracted from the cortex $(\mathrm{C})$ and hippocampus $(\mathrm{H})$ of control and epileptic animals. (A) The PCR products were run on $1 \%$ agarose gel. MRP1 (upper band, $404 \mathrm{bp}$ ) and $\beta$-actin (lower band, $241 \mathrm{bp}$ ) are shown. (B) Semi-quantitative analysis of the gel after densitometry. The expression of MRP1 was normalized and represented as a ratio of MRP1/ $\beta$-actin ( $n=8) .{ }^{\mathrm{b}} P<0.05$ compared with the control group.

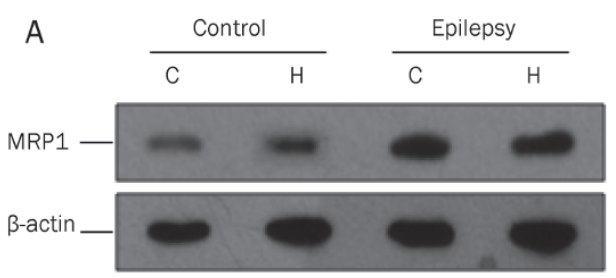

B

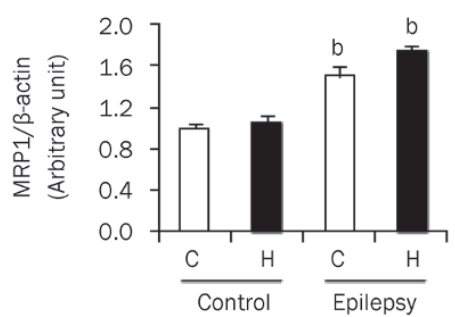

Figure 2. MRP1 protein expression in the brain. MRP1 protein was detected by Western blot. Total protein was extracted from the cortex (C) and hippocampus (H) of control and epileptic animals. (A) The total protein was separated on SDS-PAGE. MRP1 and $\beta$-actin were detected using specific antibodies. (B) Quantitative analysis of the gel after densitometry. The expression of MRP1 was normalized to $\beta$-actin $(n=8)$. ${ }^{\mathrm{b}} \mathrm{P}<0.05$ compared with the control group.

ure $3 \mathrm{~A}$ and $3 \mathrm{~B})$. In the epileptic brain, there was an increase in the number of immunoreactive cells in the cortex (Figure 3C and 3D), but the distribution and morphology of MRP1positive cells were similar to those in the control brain. After quantitative analysis, we found that the number of MRP1positive cells in the cortex was 2 times higher in the epileptic brain than that observed in the normal control brain (Figure 


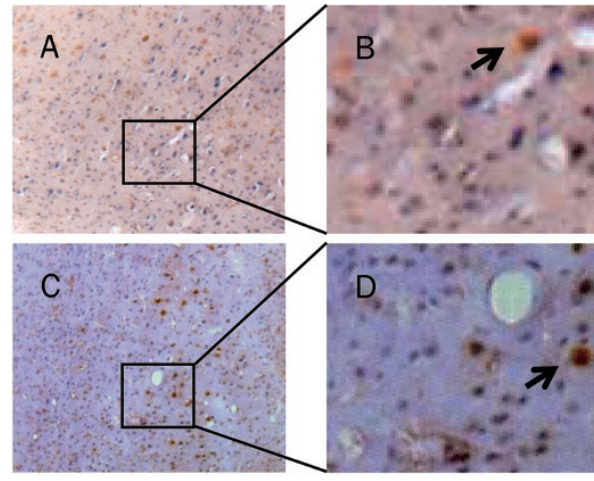

$\mathrm{E}$

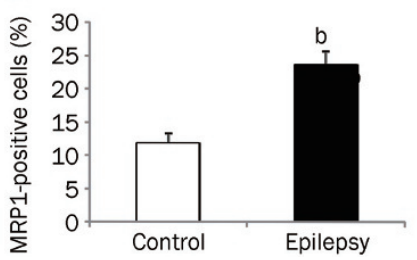

Figure 3. MRP1 expression in the cortex of rat brain. MRP1-positive cells (brown staining) were detected by immunohistochemistry. (A) Control group (×100); (B) Control group (enlarged from A); (C) Epilepsy group (×100); (D) Epilepsy group (enlarged from C). Arrow indicates a positive cell. (E) Quantitative analysis of immunohistochemistry. MRP1-positive cells were counted and represented as a percentage of total cells. At least 3 slices from each brain were measured $(n=8) .{ }^{b} P<0.05$ compared with the control group.

$3 \mathrm{E})$. In the hippocampus of the control brain, there were less MRP1-positive cells (Figure 4A and 4B). However, there were more MRP1-positive cells in the hippocampus of the epileptic brain, including in the dentate gyrus, CA1, CA2, and CA3 areas (Figure 4C and 4D). Quantitative analysis showed that the number of MRP1-positive cells in the hippocampus of the epileptic brain was significantly higher than that in the control brains (Figure $4 \mathrm{E}, P<0.05$ ).

Inhibition of MRP1 increased the concentrations of antiepileptic drugs in the cortical extracellular fluid

To examine the concentrations of antiepileptic drugs in the cortical extracellular fluid, we applied the amygdale kindling model, followed by microdialysis and HPLC analyses. To normalize the permeability of the drug through the semipermeable membrane, we first determined the recovery rate of two antiepileptic drugs, PHT and CBZ. We found that the recovery rate of PHT through the semipermeable membrane was $17.7 \% \pm 3.7 \%$, whereas the recovery rate of $\mathrm{CBZ}$ was $14.2 \% \pm 3.1 \%$.

Because the serum concentration of a drug after administration is an indicator of drug stability, first we measured the serum concentration of PHT after intraperitoneal injection. We found that the serum concentration of PHT quickly peaked $30 \mathrm{~min}$ after injection of $50 \mathrm{mg} / \mathrm{kg}$ PHT (Figure 5A). The range of the serum concentration of PHT at 15-180 min post-injection was $12.29-17.35 \mu \mathrm{g} / \mathrm{mL}$. Similarly to PHT, we
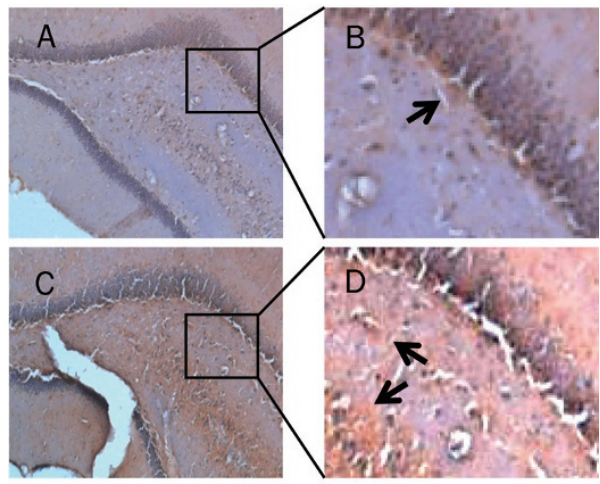

$\mathrm{E}$

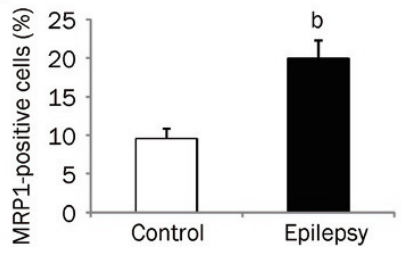

Figure 4. MRP1 expression in the hippocampus of rat brain. MRP1positive cells (brown staining) were detected by immunohistochemistry: (A) Control group (×100); (B) Control group (enlarged from A); (C) Epilepsy group (×100); (D) Epilepsy group (enlarged from C). Arrow indicates a positive cell. (E) Quantitative analysis of immunohistochemistry. MRP1positive cells were counted and represented as a percentage of total cells. At least 3 slices from each brain were measured $(n=8) .{ }^{b} P<0.05$ compared with the control group.
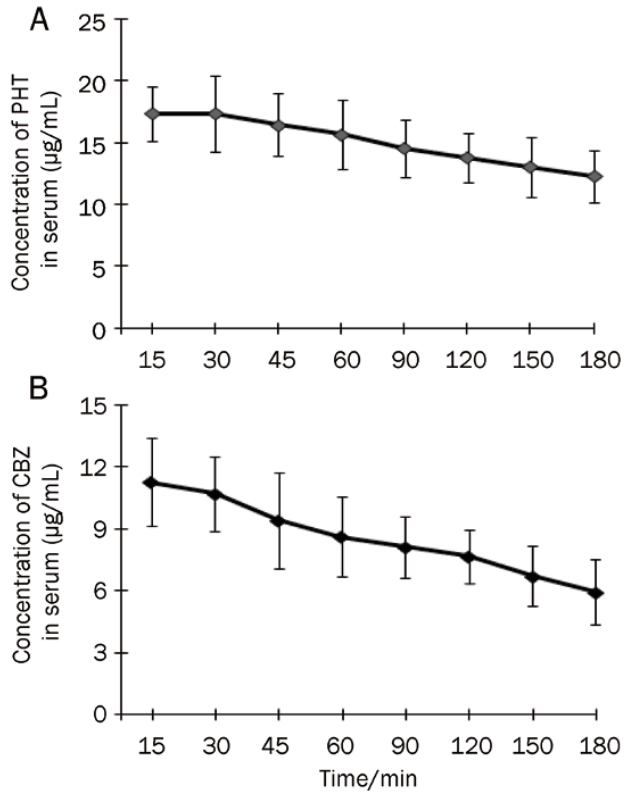

Figure 5. Serum concentrations of phenytoin and carbamazepine. The concentrations of AEDs after PHT and CBZ treatment were measured by HPLC. Serum concentrations of PHT $(A)$ and $C B Z(B)$ were measured at the times indicated (post-injection). The highest concentrations of PHT and CBZ were at $30 \mathrm{~min}$ and $15 \mathrm{~min}$, respectively, after drug injection $(n=8)$.

measured the serum concentration of CBZ after intraperitoneal injection. Administration of CBZ reached a maximum concen- 
tration $(11.26 \pm 2.12 \mu \mathrm{g} / \mathrm{mL})$ at $15 \mathrm{~min}$, and gradually decreased thereafter (Figure 5B). The range of CBZ between 15-180 min post-injection was $5.94-11.26 \mu \mathrm{g} / \mathrm{mL}$. Next, we determined the concentrations of PHT and CBZ in the cortical extracellular fluid after it passed through the blood-brain barrier. In normal rats, both PHT and CBZ can quickly pass through the bloodbrain barrier into the brain tissue, as indicated by the fact that the drugs were detected in the cortical extracellular fluid 15 min after administration. The highest concentrations of PHT and CBZ in the cortical extracellular fluid were $1.24 \pm 0.23 \mu \mathrm{g} /$ $\mathrm{mL}$ at $60 \mathrm{~min}$ post-injection and $1.52 \pm 0.22 \mu \mathrm{g} / \mathrm{mL}$ at $90 \mathrm{~min}$ post-injection, respectively (Figure 6A and 6B). Concentrations of PHT and CBZ ranged from 0.59-1.24 $\mu \mathrm{g} / \mathrm{mL}$ (PHT) and $0.71-1.52 \mu \mathrm{g} / \mathrm{mL}$ (CBZ) over the 15-180 min after injection. The ratio for PHT concentration in the cortical extracellular fluid to that in the serum was $3 \%-8 \%$, whereas the ratio for CBZ was $6 \%-19 \%$. In epileptic rats, PHT in the cortical extracellular fluid was detectable at $15 \mathrm{~min}$, peaked at $60 \mathrm{~min}$, and gradually decreased thereafter. By comparison, CBZ was also detectable at $15 \mathrm{~min}$, but peaked at $90 \mathrm{~min}$. The trend of fluctuation in the cortical extracellular fluid of epileptic brains was similar to that of the normal control brains. However, compared with normal controls, PHT and CBZ in the cortical extracellular fluid of epileptic animals were significantly lower at each time point. Finally, we administered probenecid, an inhibitor of MRP1, and measured the PHT and CBZ concentrations again. The administration of probenecid did not change the trend of fluctuation of PHT and CBZ, but significantly

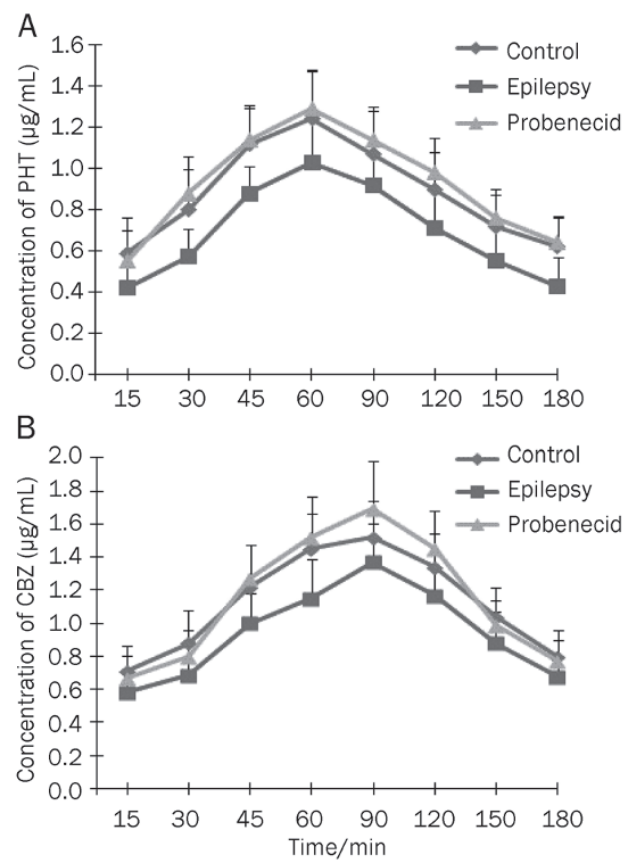

Figure 6. Concentrations of phenytoin and carbamazepine in the cortical extracellular fluid. The concentrations of AEDs were measured by HPLC. The concentrations of PHT (A) and CBZ (B) in the cortical extracellular fluid after intraperitoneal injection are shown $(n=8)$. Control, electrodeimplanted control rat; Epilepsy, epileptic rat; Probenecid, epileptic rat with probenecid pre-treatment. increased the concentrations of PHT and CBZ in the cortical extracellular fluid of epileptic animals (Figure 6A and 6B). The concentrations of PHT and CBZ in epilepsy brains after probenecid treatment was similar to that in normal control, indicating that the inhibition of MRP1 can restore the accumulation of AEDs in the cortical extracellular fluid of epileptic brains.

\section{Discussion}

MRP1 is a glycoprotein known as ABCC1 and belongs to the ATP-binding protein superfamily ${ }^{[8]}$. This molecule has three membrane-spanning domains and two nucleotide-binding domains, which function as the ATP-dependent drug pump that effluxes drugs and organic anions across the plasma membrane ${ }^{[4,9]}$. Recent studies show that MRP1 is encoded by a gene located in chromosome $16 \mathrm{p} 13.1^{[10]}$ and is involved in chemotherapy drug resistance $^{[0]}$. The current study explored the role of MRP1 in mediating two AEDs, phenytoin and carbamazepine, in refractory epilepsy.

MRP1 is an important multidrug transporter in the brain $^{[11,12]}$, despite the fact that it is mainly distributed in the choroid plexus and ependymal epithelial cells and is found in lower levels in neurons and glial cells in the normal brain $^{[13,14]}$. This trend is consistent with our observation that MRP1 mRNA and protein levels were low in the cortex and hippocampus of normal rats. It has been shown that MRP1 is involved in the blood-brain barrier (BBB) and blood-cerebrospinal fluid barrier function ${ }^{[15]}$, which prevent the influx of exogenous toxic substances into the brain tissue and remove excess brain metabolites to maintain a stable brain environment. In the normal condition, these low levels of MPR1 are sufficient for brain homeostasis.

However, in pathological conditions of the brain, such as refractory epilepsy and glioma, the levels of MRP1 are high $^{[14,16]}$. Our study demonstrated that the expression of MRP1 in the refractory epileptic brain was up-regulated. Compared with electrode-implanted control rats, the number of MRP1-positive cells was significantly increased in the cortex and hippocampus, especially among neurons and glial cells, after amygdale kindling. This result suggests that repeated epileptic seizures may have induced an increase in MRP1 expression. MRP1 has been found in brain lesions of surgically treated patients with dysembryoplastic neuroepithelial tumors, focal cortical dysplasia, and hippocampal sclerosis ${ }^{[17]}$. MRP1 has also been found to be expressed in astrocytes, a sub-type of glial cells, at lesion sites ${ }^{[18]}$. In addition, several studies have shown that chemical carcinogens and chemotherapeutic agents, such as cisplatin, barbiturates, as well as the antibiotic rifampicin can increase the expression of $\mathrm{MRP1}^{[19]}$. Our findings are in agreement with the recent data showing that the overexpression of MRP1 causes resistance to antiepileptic drugs ${ }^{[20,21]}$. Overexpressing human MRP1 protein in neurons of a Drosophila genetic seizure model, bang senseless (bss) mutants, blocked the attenuation of seizure behavior by PHT administration. Moreover, overexpression of MRP1 in neurons increased the tolerance of bss flies to high doses of 
$\mathrm{PHT}^{[20]}$.

MRP1 is not only located in the cell membrane but also present in the endoplasmic reticulum and Golgi apparatus, suggesting that in addition to its function as an efflux pump, MRP1 can also isolate drugs in cells and inhibit their ability to directly bind to their target, resulting in drug resistance. MRP1 is able to pump AEDs out of the brain tissue or cells, leading to a decrease in the concentrations of AEDs in the brain extracellular fluid, cytoplasm, and even organelles ${ }^{[13]}$, which may account for multidrug resistance in refractory epilepsy. Our studies confirmed that the decrease of PHT and CBZ concentrations in the cortical extracellular fluid was associated with the upregulation of MRP1 in the epileptic brain in vivo. A recent in vitro study that used a concentration equilibrium transport assay showed that common AEDs, including PHT and CBZ, are not substrates for MRP1, MRP2 or MRP5 in MRP-transfected kidney cell lines ${ }^{[22]}$. However, the influence of high drug permeability is minimized by this assay. Only in vivo techniques may ultimately prove whether AEDs are substrates for human MRPs.

The mechanism by which MRP1 is highly expressed in the epileptic brain is unclear. It may be a mechanism similar to that of PGP in the epileptic brain, although MRP1 is only 15\% homologous in amino acid sequence to PGP. Their substrates are close and have a considerable overlap ${ }^{[23]}$. However, MRP1 is less specific to the substrates. Several studies confirmed that MRP1 is an ATP energy-dependent transmembrane protein with a glutathione (GSH)-S-conjugate operating pump that requires the participation of GSH to transport conjugated substrates ${ }^{[18,24]}$. Mrp $1^{(--)}$astrocytes have a $50 \%$ higher specific GSH content than wild-type or Mrp5 $5^{(-/)}$cells. In wildtype astrocytes, MRP1 can mediate $60 \%$ of the GSH export ${ }^{[18]}$. MRPs contain two binding sites, one with low GSH affinity and another with high GSH affinity. Therefore, there are two transport mechanisms. Nonconjugated organic anions can directly be transported out of cells, whereas the transportation of cationic or neutral charge molecules requires $\mathrm{GSH}^{[25]}$.

Probenecid has been considered to be an effective antagonist of MRP1. A previous study showed that probenecid significantly increased the concentration of sodium valproate in dog cerebrospinal fluid ${ }^{[26]}$. In the current study, PHT and CBZ were recognized by MRP1, and thus, the ability of the drugs to pass through the BBB was impaired. By inhibiting MRP1 with probenecid, we observed that the concentrations of PHT and CBZ were restored in the cortical extracellular fluid in rats with refractory epilepsy. Probenecid can also inhibit the function of MRP2, another transporter of AEDs in the brain, found mainly in the luminal surface of brain capillary endothelium. The concentration of PHT in the brain of Mrp2-deficient rats was significantly higher than in the wild-type rats ${ }^{[27]}$, indicating that MRP2 substantially contributes to BBB function. MRP1 and MRP5 are expressed in normal human astrocytes and endothelial cells. High levels of MRP1, moderate levels of MRP5, and low levels of MRP3 were found in tumor cells of malignant glioma patients, suggesting that MRP1 may be responsible for tumor cells' resistance to chemotherapy in the brain $^{[11]}$. MRP1 can cause resistance to arsenite, while MRP2 can result in resistance to cisplatin ${ }^{[9]}$. MRP1, but not MRP5, mediates export of glutathione and glutathione disulfide in brain astrocytes ${ }^{[18]}$. Presently there is no report of the effect of MRP3 on PHT and CBZ. The current study did not rule out the involvement of MRP2, MRP3, and MRP5. Using specific inhibitors of MRPs or knocking-down individual MRPs may be applied in future experiments.

In addition to PHT and CBZ, other xenobiotics and endobiotics may also be the substrates of $\mathrm{MRP}^{[28]}$. Although changes in the properties of the targets of AEDs resulted in the decrease of its sensitivity, they do not explain the multidrug resistance. The loss of the ability of AEDs to cross the BBB may be attributed to the overexpression of multi-drug transporters, such as MRP1, leading to the decrease of the drug concentrations in the cortical extracellular fluid and the impairment of the access of AEDs to their targets in the brain cells of epileptic patients.

In conclusion, our data demonstrated that MRP1 was expressed in the normal rat brain but up-regulated in the epileptic rat brain. The increase of MRP1 reduced the concentrations of AEDs, whereas the inhibition of MRP1 restored the concentrations of AEDs in the cortical extracellular fluid, suggesting that MRP1 is a key transporter involved in the mechanism underlying brain cell resistance to AEDs in refractory epilepsy.

\section{Acknowledgements}

This work was supported by grants from the Natural Science Foundation of Shanghai (09ZR1405500) and the Shanghai Municipal Health Bureau (2008-08) to Yin-hui CHEN and a start-up fund of research from the Jinshan Hospital of Fudan University to Guoxiong XU (2012-2).

\section{Author contribution}

Ying-hui CHEN contributed to the development of project and the design and performance of experiments. Cui-cui WANG performed Western blot and RT-PCR. Xia XIAO performed microdialysis. Li WEI participated in the data analysis and wrote the manuscript. Guoxiong XU contributed to the data analysis, the generation of figures and the writing of the manuscript. All authors read and approved the final manuscript.

\section{References}

1 Siddiqui A, Kerb R, Weale ME, Brinkmann U, Smith A, Goldstein $\mathrm{DB}$, et al. Association of multidrug resistance in epilepsy with a polymorphism in the drug-transporter gene ABCB1. N Engl J Med 2003; 348: 1442-8.

2 Lazarowski A, Czornyj L. Potential role of multidrug resistant proteins in refractory epilepsy and antiepileptic drugs interactions. Drug Metabol Drug Interact 2011; 26: 21-6.

3 Baguley BC. Multiple drug resistance mechanisms in cancer. Mol Biotechnol 2010; 46: 308-16.

4 Hipfner DR, Deeley RG, Cole SP. Structural, mechanistic and clinical aspects of MRP1. Biochim Biophys Acta 1999; 1461: 359-76.

5 Morimoto K, Goddard GV. Seizure-triggering mechanism in the kindling model of epilepsy: I. EEG changes during stimulation from 
the site of stimulation. Jpn J Psychiatry Neurol 1988; 42: 618-9.

6 Racine RJ. Modification of seizure activity by electrical stimulation. II. Motor seizure. Electroencephalogr Clin Neurophysiol 1972; 32: 281-94

7 Walker MC, Alavijeh MS, Shorvon SD, Patsalos PN. Microdialysis study of the neuropharmacokinetics of phenytoin in rat hippocampus and frontal cortex. Epilepsia 1996; 37: 421-7.

8 Iram SH, Cole SP. Expression and function of human MRP1 (ABCC1) is dependent on amino acids in cytoplasmic loop 5 and its interface with nucleotide binding domain 2. J Biol Chem 2011; 286: 7202-13.

9 Borst P, Evers R, Kool M, Wijnholds J. A family of drug transporters: the multidrug resistance-associated proteins. J Natl Cancer Inst 2000; 92: 1295-302.

10 Perdu J, Germain DP. Identification of novel polymorphisms in the pM5 and MRP1 (ABCC1) genes at locus 16p13.1 and exclusion of both genes as responsible for pseudoxanthoma elasticum. Hum Mutat 2001; 17: 74-5.

11 Calatozzolo C, Gelati M, Ciusani E, Sciacca FL, Pollo B, Cajola L, et al. Expression of drug resistance proteins P-gp, MRP1, MRP3, MRP5 and GST-pi in human glioma. J Neurooncol 2005; 74: 113-21.

12 Kubota H, Ishihara H, Langmann T, Schmitz G, Stieger B, Wieser $\mathrm{HG}$, et al. Distribution and functional activity of P-glycoprotein and multidrug resistance-associated proteins in human brain microvascular endothelial cells in hippocampal sclerosis. Epilepsy Res 2006; 68: 213-28.

13 Lazarowski A, Lubieniecki F, Camarero S, Pomata H, Bartuluchi M, Sevlever G, et al. Multidrug resistance proteins in tuberous sclerosis and refractory epilepsy. Pediatr Neurol 2004; 30: 102-6.

14 Dombrowski SM, Desai SY, Marroni M, Cucullo L, Goodrich K, Bingaman W, et al. Overexpression of multiple drug resistance genes in endothelial cells from patients with refractory epilepsy. Epilepsia 2001; 42: 1501-6.

15 Loscher W, Potschka H. Blood-brain barrier active efflux transporters: ATP-binding cassette gene family. NeuroRx 2005; 2: 86-98.

16 Bronger H, Konig J, Kopplow K, Steiner HH, Ahmadi R, Herold-Mende C, et al. ABCC drug efflux pumps and organic anion uptake transporters in human gliomas and the blood-tumor barrier. Cancer Res 2005; 65: 11419-28.

17 Sisodiya SM, Lin WR, Harding BN, Squier MV, Thom M. Drug resistance in epilepsy: expression of drug resistance proteins in common causes of refractory epilepsy. Brain 2002; 125: 22-31.

18 Minich T, Riemer J, Schulz JB, Wielinga P, Wijnholds J, Dringen R. The multidrug resistance protein 1 (Mrp1), but not Mrp5, mediates export of glutathione and glutathione disulfide from brain astrocytes. J Neurochem 2006; 97: 373-84.

19 Schrenk D, Baus PR, Ermel N, Klein C, Vorderstemann B, Kauffmann HM. Up-regulation of transporters of the MRP family by drugs and toxins. Toxicol Lett 2001; 120: 51-7.

20 Bao GS, Wang WA, Wang TZ, Huang JK, He H, Liu Z, et al. Overexpression of human MRP1 in neurons causes resistance to antiepileptic drugs in Drosophila seizure mutants. J Neurogenet 2011; 25: 201-6.

21 Kuteykin-Teplyakov K, Brandt C, Hoffmann K, Loscher W. Complex time-dependent alterations in the brain expression of different drug efflux transporter genes after status epilepticus. Epilepsia 2009; 50: 887-97.

22 Luna-Tortos C, Fedrowitz M, Loscher W. Evaluation of transport of common antiepileptic drugs by human multidrug resistanceassociated proteins (MRP1, 2 and 5) that are overexpressed in pharmacoresistant epilepsy. Neuropharmacology 2010; 58: 101932.

23 Lautier D, Canitrot Y, Deeley RG, Cole SP. Multidrug resistance mediated by the multidrug resistance protein (MRP) gene. Biochem Pharmacol 1996; 52: 967-77.

24 Nunoya K, Grant CE, Zhang D, Cole SP, Deeley RG. Molecular cloning and pharmacological characterization of rat multidrug resistance protein 1 (mrp1). Drug Metab Dispos 2003; 31: 1016-26.

25 Salerno M, Garnier-Suillerot A. Kinetics of glutathione and daunorubicin efflux from multidrug resistance protein overexpressing small-cell lung cancer cells. Eur J Pharmacol 2001; 421: 1-9.

26 Frey $\mathrm{HH}$, Loscher W. Distribution of valproate across the interface between blood and cerebrospinal fluid. Neuropharmacology 1978; 17: $637-42$

27 Potschka H, Fedrowitz M, Loscher W. Multidrug resistance protein MRP2 contributes to blood-brain barrier function and restricts antiepileptic drug activity. J Pharmacol Exp Ther 2003; 306: 124-31.

28 Munoz M, Henderson M, Haber M, Norris M. Role of the MRP1/ ABCC1 multidrug transporter protein in cancer. IUBMB Life 2007; 59: 752-7. 